\title{
A Review on MVdc Collection Systems for High Power Offshore Wind Farms
}

\author{
Gayan Abeynayake, Gen Li, Jun Liang \\ School of Engineering, Cardiff University, Cardiff, \\ United Kingdom \\ AbeynayakePA@cardiff.ac.uk
}

\author{
Nicolaos A. Cutululis \\ Department of Wind Energy, \\ Technical University of Denmark, \\ Denmark
}

\begin{abstract}
Recently, offshore renewable technologies have shown a rapid growth with the advancement in technology together with reduction of capital investment per unit size. This movement is further catalyzed by various government policy decisions and new set targets on renewable energy (RE) production. Due to higher wind speed, limited land availability and flexibility of transporting very large structures (with the increased size of a single wind turbine) offshore wind power plants provide commendable benefits over onshore wind plants. At present, only medium voltage ac is used in the offshore wind collection systems. However, the use of medium voltage direct current (MVdc) at the collection level is becoming more attractive due to advantages like lower systems losses, zero reactive power compensation and flexibility of power flow control compared to traditional ac collection systems. This paper intends to review opportunities and key challenges associated with the emerging MVdc technology for offshore wind collection systems. Attention is given to the DC wind turbine concept and selecting a suitable MVdc collection system topology with appropriate control modes.
\end{abstract}

Index Terms--Medium voltage dc (MVdc), offshore wind, wind power collection systems, dc wind turbine (dcWT), research challenges.

\section{INTRODUCTION}

The global trend towards power generation through renewable energy (RE) sources have been increasing over the last few decades due to significant advantages over coal- and fossil fuel- based power plants. The use of fossil fuels to meet the global energy demand has major drawbacks such as contribution to global warming and depletion of resources over time. According to [1], the use of coal for energy production in the global energy mix is set to decline by almost $80 \%$ from 2017 to 2040 under sustainable development scenario. In the context of Europe, by 2030 it is expected to produce at least $32 \%$ of total energy consumption from renewable energy and $40 \%$ cut in greenhouse gas emissions compared with 1990 [2].

The above issues have given rise to the promotion of RE solutions such as wind, solar PV, small hydro, biomass, geothermal, wave and tidal power. For example, by end of 2017, the regional wind power production capacity was distributed as follows: 177.5 GW Europe; 228.6 GW Asia; 105.3 GW North America; 17.8 GW Latin America; 4.5 GW Africa; and 5.1 GW Pacific regions [3]. The cumulative capacity of wind turbine

This work received funding from the European Union's Horizon 2020 research and innovation programme under the Marie Sklodowska-Curie grant agreement no. 765585 , project title InnoDC.

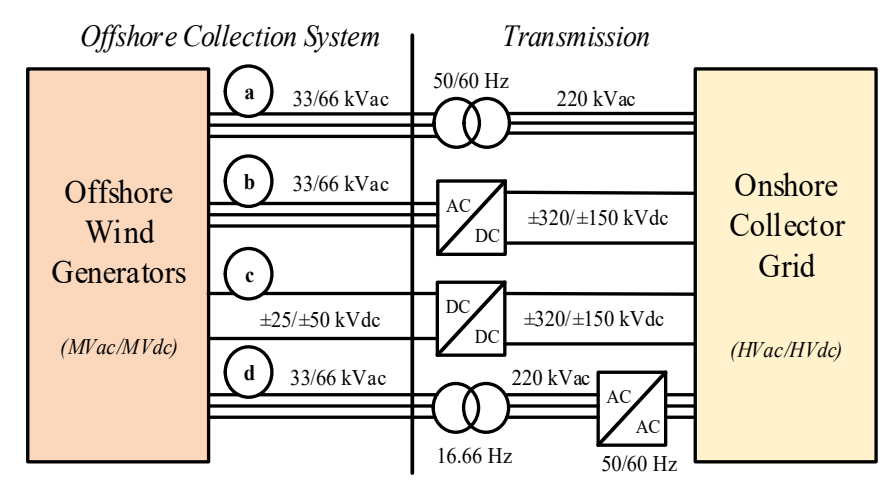

Fig. 1. Different OWPP Collection System Options: (a) MVac collection with HVac transmission; (b) MVac collection with HVdc transmission; (c) MVdc collection with HVdc transmission; (d) Low frequency MVac collection and HVac transmission.

(WT) installation was increased by $9.1 \%$ by the end of 2018 [4]. These figures are forecasted to be risen in the future with the advancement of wind power technology, incentives from governments and reduction in capital investment per MW. Offshore wind technology provides added benefits such as higher energy yield and lesser public disturbance over its counterpart onshore wind technology. At present, Europe is the leader in offshore wind power plant (OWPP) development. As at 2018 , total globally installed offshore wind capacity is 22,045 MW and more than $80 \%$ is concentrated to Europe [5]. Within Europe, the UK has the largest amount of offshore wind capacity with $44 \%$ of all installations. Some major projects such as Walney Extension, London Array, Norfolk Vanguard and Norfolk Boreas are notable [6].

Different OWPP collection system options with transmission technologies are shown in Fig. 1. At present, all existing offshore wind parks feature an internal ac network with either ac or dc transmission to onshore power grids. The advantage of using the state-of-the-art ac technology from the onshore power grid is obvious in terms of know-how, experience, and, to a certain extent, cost [7]. However, as the OWPP distance to onshore grid increases (where wind conditions are superior) the ac cable transmission may become problematic due to high capacitive cable charging. Thanks to the development of voltage source converter (VSC), particularly the modular multilevel converters, the high voltage 


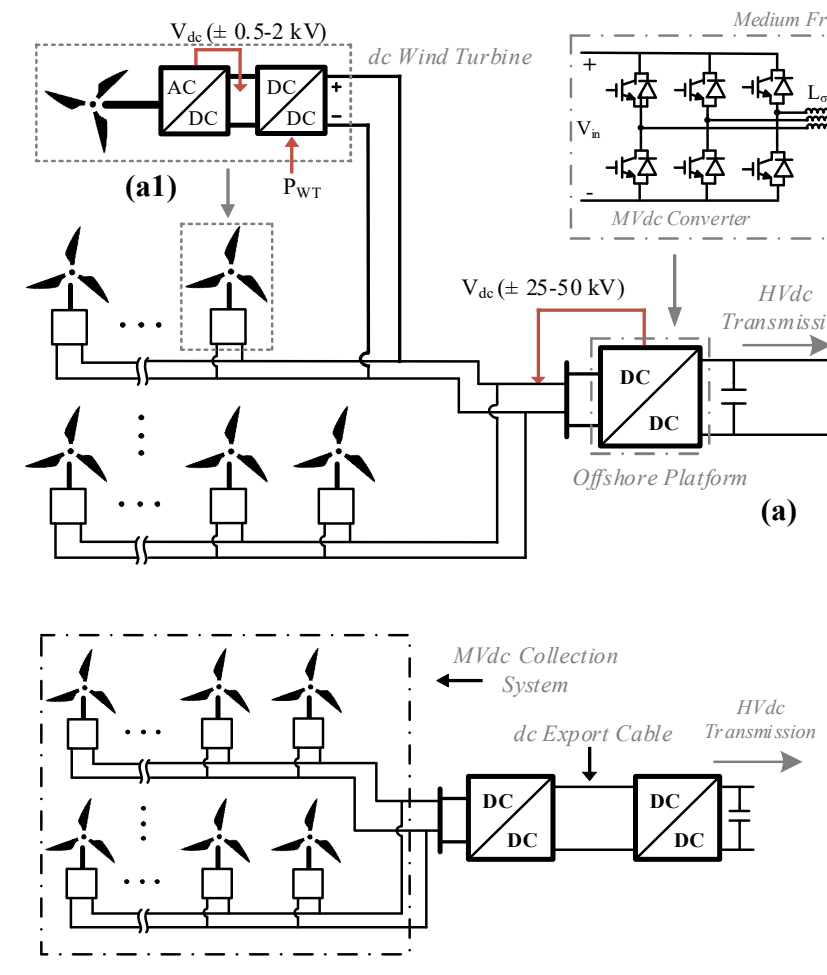

(c)

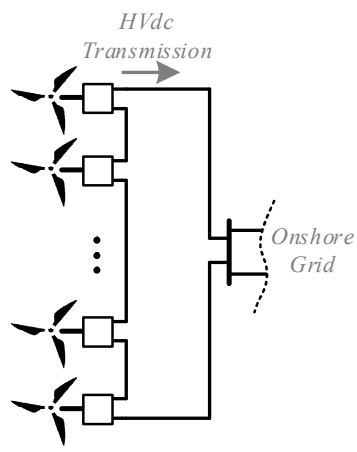

(b)

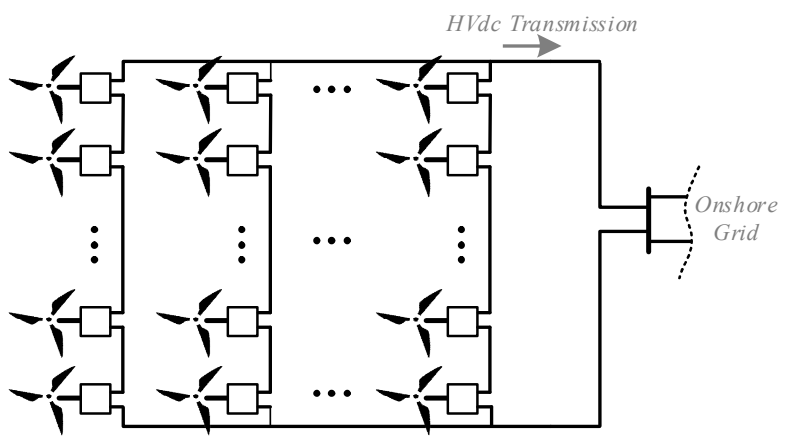

(d)

Fig. 2. Different MVdc collection systems: (a) Shunt topology -1; (b) Series topology; (c) Shunt topology -2; (d) Series-parallel topology.

direct current (HVdc) connection can be an alternative for longdistance offshore power integration [8]-[10]. Bard-1, $400 \mathrm{MW}$ wind park $130 \mathrm{~km}$ off the coast of Germany, is the first offshore wind park connected through a HVdc transmission link to the main grid [11]. Due to additional benefits such as lower losses, zero reactive power compensation, elimination of $50 / 60 \mathrm{~Hz}$ bulky transformers, lightweight and reduction in the size of power electronic converters MVdc technology will be a promising option for future OWPP collection systems [12][14]. Thus, this paper attempts to review suitable MVdc collection systems and dcWT topologies for future large scale OWPPs.

The rest of the paper is organized as follows. Section II. discusses feasible MVdc collection system topologies with different control modes. The key component in an all-dc OWPP; the dc wind turbine (dcWT) is discussed in Section III. Section IV. highlights existing technical barriers of MVdc collection systems. Finally, Section V. summarizes the main conclusions from this paper with some important future research directives.

\section{DIFFERENT DC COLLECTION SYSTEM TOPOLOGIES AND CONTROL}

Irrespective of the technology used identifying a suitable offshore wind farm collection system is important. The main factors to be considered are power losses and capital investment. In [15]-[16], it has shown that the use of MVdc collection systems for energy harvesting from OWPPs are economically beneficial over existing MVac collection systems. However, other technical challenges such a) protection against dc faults; b) stability and control under wake effect and other system disturbances; c) availability under scheduled maintenances; d) reliability of the overall collection system (with the use of a large number of power electronic devices) need to be addressed. Special attention should be given to reliability and maintainability upon wind turbine and collection system component failures under marine harsh weather environment [17].

The primary requirement of a high power MVdc collection system is the development of a dcWT which is still at incubator research stage. The collection system comprises of several MVdc clusters (typical voltage range between $\pm 25 \mathrm{kV}$ to \pm 50 $\mathrm{kV}$ ) which will be collected by one or several offshore collection system platforms (depending on the distance to shore) and export to the onshore grid via HVdc transmission. Different dc collection system configurations have been proposed in the literature. Primarily it can be categorized into shunt- and series- topology [7], [18] and others are variants of these two basic topologies as shown in Fig. 2.

\section{A. Collection System Topologies}

\section{a) Shunt configurations}

Depending on the distance to the intermediate offshore platform, there may be different shunt configurations as shown in Figs. 2(a) and (c). Shunt topology-1 can be used if the wind turbine clusters are located close to centralized collection platform and all the MVdc cables directly connect to the offshore HVdc converter platform. Shunt topology-2 is very similar to topology-1 except the additional $\mathrm{dc} / \mathrm{dc}$ converter. This topology can be used if clusters are located far away from the onshore grid and power transmission requires an additional intermediate step to boost the voltage before $\mathrm{HVdc}$ 
transmissions. However, reliability of these two configurations at the cluster level can be increased by introducing additional $\mathrm{dc} / \mathrm{dc}$ converters at the expense of capital investment. The interarray cable network can be configured as single-sided radial feeder, bifurcated-radial feeder, single-sided or double-sided ring feeder as discussed in [12].

\section{b) Series and series-parallel configurations}

The main advantage of series and series-parallel (SP) topologies is the elimination of the offshore collector platform. Induvial dc wind turbines are connected in series in such a way that the voltage is built up to transmission levels. Theoretically, the current in the series connected wind array needs to be the same. If all the WTs have the same rated output, to satisfy this condition they must produce the same output power. However, in a condition where WTs in the same array produce different power outputs, the WTs operating below the average wind speed must increase its terminal voltage (and vice versa for WTs operating above the average) to maintain the nominal transmission voltage.

If the permissible voltage limit exceeds, these WTs will be forced to shut down which may result in the total failure of the entire string. This is the major drawback of this topology. To address this issue in SP topology a novel configuration called "matrix topology' was introduced in [19] which uses intermediate switches between adjacent strings. However, with the increase of number of parallel branches a sophisticated control scheme is necessary to switch between adjacent wind arrays in SP topology.

\section{B. Offshore Collection Platforms}

In an all-dc OWPP, core of the offshore collection platform is the $\mathrm{dc} / \mathrm{dc}$ converter which boost the MVdc voltage level to $\mathrm{HVdc}$ transmission voltage level. There are many variants of $\mathrm{dc} / \mathrm{dc}$ converter topologies which are suitable for high power DC grids [20]. The isolated $\mathrm{dc} / \mathrm{dc}$ converter which uses a medium frequency transformer (operating at $1-10 \mathrm{kHz}$ ) is considered here. It operates on phase shift control mode [single active bridge (SAB) and dual active bridge (DAB)]. Fig. 2(a2). shows the configuration of a three-phase variant of the DAB proposed in [21]. As expressed in (1) and (2) (for single-phase and three-phase) the power flow can be varied by regulating the phase shift. In (1) and (2), $V_{\text {in }}$ and $V_{\text {out }}$ represent the input and output dc voltages, $\phi-$ the phase shift, $n$ - transformer turns ratio, $f_{s}$ - switching frequency and $L_{\sigma}$ - leakage inductance.

$$
\begin{aligned}
& P_{D A B(1 \phi)}=\frac{V_{\text {in }} V_{\text {out }}}{n 2 \pi f_{S} L_{\sigma}} \phi\left(\frac{1-\phi}{\pi}\right) \quad ; 0 \leq \phi<\frac{\pi}{2} \\
& P_{D A B(3 \phi)}=\frac{n V_{\text {in }} V_{\text {out }}}{2 \pi f_{S} L_{\sigma}} \phi\left(\frac{2}{3}-\frac{\phi}{2 \pi}\right) ; 0 \leq \phi<\frac{\pi}{3}
\end{aligned}
$$

However, in the shunt topology-1 of Fig. 2(a), the control objective of the offshore collection platform is to maintain the collection system MVdc voltage. Once the HVdc voltage is fixed by the onshore VSC, collection system MVdc voltage can be regulated with closed-loop control as illustrated in [22].

\section{Control of MVdc OWPP Collection Systems}

In order to harmonize with the onshore grid requirements and harness maximum power from the offshore wind plant, the optimum controlling of entire OWPP cluster is important. Thus, in a pure dc-based wind farm, maintaining the specific dc voltages at different stages of the OWPP collection system is essential. However, no frequency and reactive power control is required unlike in MVac collection systems.

The control responsibilities of different converters starting from the dcWT level up to onshore converters are illustrated using Fig. 1(a). Respective arrow in the figure indicates the dc voltage control requirements from the specific converter. For instance, the MVdc voltage is regulated by offshore collection system dc/dc converter and HVdc voltage by onshore VSC. The power output from wind turbines $\left(\mathrm{P}_{\mathrm{WT}}\right)$ is controlled by the wind turbine $\mathrm{dc} / \mathrm{dc}$ converter which is discussed in the next section.

\section{DC WIND TURBINES}

Before discussing the dcWT concept, it is worth to consider the exiting ac wind turbine technology (acWT) currently deploying at OWPPs. At present, majority of offshore WTs are constructed with variable speed permanent magnet synchronous generators (PMSG) due to benefits such as compact in size, no requirement of external power supply for magnetic field excitation, robust construction and less maintenance compared to classical asynchronous wind generators.

\section{A. PMSG based full-scale ac Wind Turbine}

In a conventional variable speed full-scale offshore wind turbine, the generator output is connected through a back-toback (ac/dc/ac) converter, with a constant frequency and voltage. At the collection level, the acWT output voltage increase up to $33 \mathrm{kVac}$ or $66 \mathrm{kVac}$ using a step-up transformer. The control schematic of a typical grid connected PMSG based full-scale acWT is shown in Fig. 3.

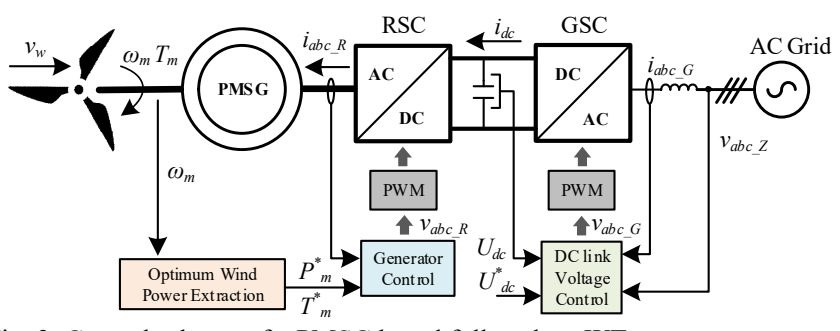

Fig. 3. Control scheme of a PMSG based full-scale acWT.

According to Fig. 3, there are three different control objectives of a full-scale wind turbine:

\section{a) Optimum wind power extraction}

It provides the necessary power $\left(\mathrm{P}^{*}{ }_{\mathrm{m}}\right)$ or torque reference $\left(\mathrm{T}^{*}{ }_{\mathrm{m}}\right)$ based on the operational points of maximum efficiency. The optimal reference value depends on the rotor speed, wind speed, and pitch angle. 


\section{b) DC link voltage control}

The grid side converter (GSC) is responsible for maintaining a constant dc link voltage and it is able to provide reactive power. Typically, the control objective is achieved by the vector control which is based on $d q$ components. This consists of an outer loop that regulates the dc voltage through a proportional integral (PI) controller and an inner loop that controls the current.

\section{c) Generator control}

The rotor side converter (RSC) controls the power extracted from the wind turbine or the mechanical torque. However, in [23] it has shown that it is possible to swap control objectives of GSC and RSC with enhanced fault ride-through and voltage support capabilities.

\section{B. DC Wind Turbines}

\section{a) Proposed Configurations}

There are several dcWT concepts discussed in the literature as illustrated in Fig. 4. They are classified according to the number of converter stages assuming state-of-the-art wind generators are employed [20].
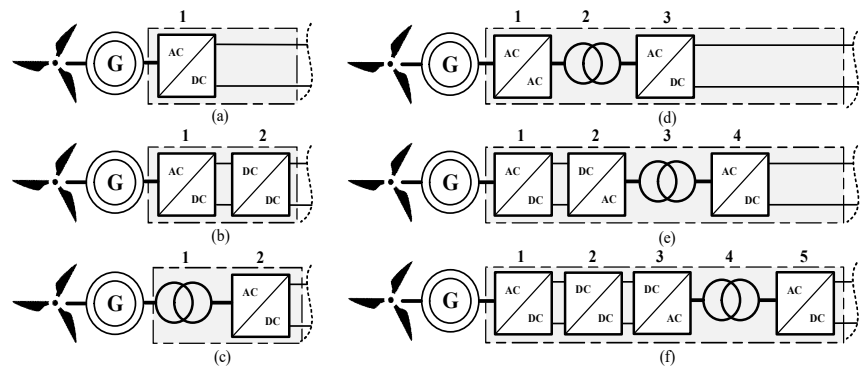

Fig. 4. Different dcWT configurations: (a) Active rectified based dcWT; (b) non-isolated dc/dc converter based dcWT; (c) ac transformer and passive rectifier based dcWT; (d) matrix converter, medium frequency transformer and passive rectifier based dcWT; (e) active rectifier and galvanic isolated $\mathrm{dc} / \mathrm{dc}$ converter based dcWT; (f) boost converter between the active rectifier and galvanic isolated DC/DC converter based dcWT.

Minimum number of components, simplicity and galvanic isolation are the important factors to be considered when selecting a suitable dcWT topology. The simplest design is the topology-(a) but the maximum output voltage is restricted by generator's nominal voltage level and it has no galvanic separation. The isolation issue in concept-(a) can be eliminated by adding a non-isolated dc/dc converter. The topology in Fig. 4(c) uses a low-frequency transformer with a passive rectifier as proposed in [24]. However, fixed speed operation and transformer saturation are the main disadvantages.

Concept-(d) deploys a matrix converter (MC) at stage-1 which is proposed in [25] with galvanic isolation. However, $\mathrm{MC}$ requires advanced control strategies to coordinate with variability of wind power which could be an interesting research topic. Concept-(e) provides galvanic isolation and uses state-of-the-art WT and active rectifier which is similar to type4 ac wind generators. In this topology, high power $\mathrm{dc} / \mathrm{dc}$ converter is the only component added to complete dcWTs. The topology-(f) proposed in [26] uses a boost converter between the active rectifier and the isolated $\mathrm{dc} / \mathrm{dc}$ converter. It assumes that the boost converter actively controls the LV side de link, while the dc/dc converter operates in an open loop. Among the topologies discussed, concept-(e) is the most suitable topology due to low technology risk.

\section{b) Suitable dc/dc converter configurations}

$\mathrm{Dc} / \mathrm{dc}$ converters need to consider as a combination of power conversion stages 2,3 and 4 of Fig. 4(e). This dc/dc converter topology can be $\mathrm{SAB}$ or $\mathrm{DAB}$ which operates on phase-shift control principle. In wind power applications, the active power flow is always unidirectional i.e. from WT side to grid side. Therefore, SAB will primarily serve our requirement. However, the major limitation of $\mathrm{SAB}$ is phase shift between primary and secondary occurs due to the leakage inductance of the medium frequency transformer [15]. Thus, to transfer large amount of power transformer leakage inductance should made very low. This problem shall eliminate with the use of DAB.

Generally, DAB is used when bi-directional power transfer is required such as in dc microgrids. However, DAB shall support for OWPP black-start by enabling reverse power flow to charge the dc-link of the dcWT. If SAB is used, there should be a stand-by diesel generator ready at all times. With severer marine weather conditions, re-fueling of diesel generators could be problematic. Thus, the use of DAB as the $\mathrm{dc} / \mathrm{dc}$ converter in topology-(e) is advantageous.

\section{c) dcWT control}

As shown in Fig. 5. the main control objectives of topology-(e) (refer together with Fig. 4(e)) are dc link voltage control and active power control. This is similar to the control of PMSG based full-scale acWT with interchange of control responsibilities as discussed in section-A. The dc link voltage is controlled by VSC connected to wind generator and power is regulated by DAB on phase-shift control mode.

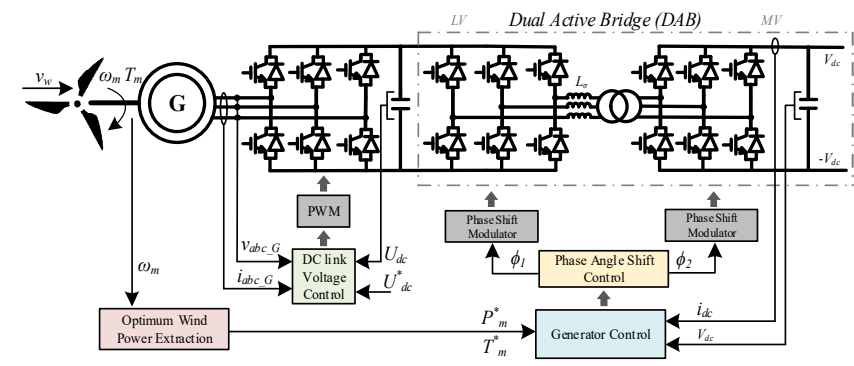

Fig. 5. Control scheme of dcWT topology-(e).

\section{Existing Challenges And Perspectives}

Unlike for MVac offshore collection systems, there is no complete standard or design guideline (which specify voltage levels, design and operational requirements, recommended systems studies and analyses, etc.) for MVdc offshore collection systems yet. However, [27] provides a detailed specification for MVdc shipboard power systems which provides some common requrements of a dc power system. Further, CIGRÉ working group C6.31 was established in order to investigate the needs and feasibility of building MVdc grids. Key challenges of an MVdc OWPP are identified as: 
- DC Fault Protection: Protection against de faults is one of the main challenges that need to be dealt with in a pure dc grid. The key requirement of a high-power dc circuit breaker (DCCB) is acting quite fast to avoid extreme high currents [28]. Off-the-shelf DCCB for MVdc shipboard power systems and traction application are not suitable due to low fault current handling capabilities. In the family of DCCBs, solid-state circuit-breaker has the overall best performance with respect to technical and reliability parameters compared to different variants of mechanical and hybrid dc breakers [29]. However, ongoing efforts should be made to improve the DCCB technology for high power MVdc grids.

- Grid Code Compliances: Irrespective of the collection system technology (MVac or MVdc) used at the offshore wind farm, it is necessary to fulfil the grid code requirements. Upon a system disturbance, dcWTs and offshore collection platform dc/dc converter must be able to control in a way that it will comply with the relevant grid code. Thus, new control strategies/methods are required to comply with stringent grid codes in an all-dc OWPP.

- Reliability: Reliability is another important factor to be considered in any engineering system. It can range from component level to the system level. A system which comprises of a large number of power electronic devices may fail at any time. Thus, reliability is a decision making factor when selecting a suitable dc collection system topology.

\section{CONCLUSION}

This paper reviewed different configurations of MVdc collection systems and dc wind turbine topologies for all-dc OWPPs. Due to advantages over classical MVac technology MVdc collection systems are suitable for future offshore wind power collection systems. Considering capital investment, controllability and reliability the variant of shunt topology is preferable for MVdc collection systems. Due to low technological risk active rectifier and galvanic isolated $\mathrm{dc} / \mathrm{dc}$ converter based dcWT is suitable. Main challenges associated with the emerging MVdc technology for offshore wind collection systems are identify as dc fault protection, strict grid code compliances and reliability. In order to overcome these issues continuous research and development efforts are required.

\section{REFERENCES}

[1] World Energy Outlook 2018, Global shifts in the energy system, [Online]. https://www.iea.org/weo/

[2] "Going Climate Neutral by 2050", A strategic long-term vision for a prosperous, competitive and climate-neutral EU Economy, 2019

[3] Global installed wind power capacity in 2017 - Regional Distribution, [Online]. http://gwec.net/global-figures/graphs/

[4] "Wind Power Capacity Worldwide reaches $597 \mathrm{GW}, 50,1 \mathrm{GW}$ added in 2018, World Wind Energy Association, Press Release, June 2019

[5] "Offshore Wind in Europe - Key trends and statistics 2018", WindEurope, 2018
[6] X. Li, G. Abeynayake, L. Yao, J. Liang, "Recent Development and Prospect of Offshore Wind Power in Europe", Journal of Global Energy Interconnection, Vol. 2 No. 2, Mar. 2019

[7] D. Van Hertem, O. Gomis-Bellmunt, and J. Liang, "HVDC Grids for Offshore and Supergrid of the Future, Wiley-IEEE Press, April 2016

[8] D. Elliott et al., "A Comparison of AC and HVDC Options for the Connection of Offshore Wind Generation in Great Britain," IEEE Trans. Power Del., vol. 31, no. 2, pp. 798-809, April 2016

[9] G. Li, J. Liang, F. Ma, C. E. Ugalde-Loo and H. Liang, "Analysis of Single-Phase-to-Ground Faults at the Valve Side of HB-MMC in HVDC Converter Stations," IEEE Trans. Ind. Electron., vol. 66, no. 3, pp. 24442453, March 2019.

[10] G. Li, W. Liu, T. Joseph, J. Liang and Z. Song, "Double-Thyristor based Protection for Valve-Side Single-Phase-to-Ground Faults in HB-MMC based Bipolar HVDC Systems," IEEE Trans. Ind. Electron., Jul. 2019.

[11] Bard Offshore [Online]. https://www.windpoweroffshore.com/bardoffshore

[12] K. Musasa, N. I. Nwulu, M. N. Gitau and R. C. Bansal, "Review on DC collection grids for offshore wind farms with high-voltage DC transmission system," IET Power Electr., vol. 10, no. 15, 2017.

[13] Lakshmanan, P., Liang, J., Jenkins, N.: 'Assessment of collection systems for HVDC connected offshore wind farms', Electr. Power Syst. Res., 2015, 129, pp. 75-82

[14] Musasa, K., Gitau, M.N., Bansal, R.C.: 'Analysis of a DC collectorbased power converter topology for an offshore wind farm', Electr. Power Compon. Syst., 2015, 43, (10), pp. 1113-1121

[15] C. Meyer, "Key components for future offshore dc grids", PhD. Thesis, RWTH, Aachen University, 2007.

[16] L. Max, "Design and control of a dc collection grid for a wind farm", PhD. Thesis, Dept. of Energy and Environment, Chalmers University of Technology, 2009.

[17] C. Zhan, C. Smith, a. Crane, a. Bullock, and D. Grieve, "DC transmission and distribution system for a large Offshore Wind Farm," 9th IET Int. Conf. AC DC Power Transm. (ACDC 2010), 2010.

[18] H. J. Bahirat, B. A. Mork and H. K. Høidalen, "Comparison of wind farm topologies for offshore applications," 2012 IEEE Power and Energy Society General Meeting, San Diego, CA, 2012, pp. 1-8.

[19] Chuangpishit, S., Tabesh, A.: 'Topology design for collector systems of offshore wind farms with pure DC power systems', IEEE Trans. Ind. Electron., 2014, 61, (1), pp. 320-328

[20] Dincan CG. "High power medium voltage DC/DC converter technology for DC wind turbines", PhD Thesis, Aalborg University, 2018.

[21] R. De Doncker, D. Divan, M. Kheraluwala, "A three-phase soft-switched high-power-density DC/DC converter for high-power applications," IEEE Trans. on Ind. Appl., vol. 27, no. 1, pp. 63-73,1991.

[22] W. Zhifu, W. Yupu, R. Yinan,"Design of closed-loop control system for a bidirectional full bridge DC/DC converter", Applied Energy, Vol. 194, 2017, pp. 617-625

[23] A. D. Hansen and G. Michalke, "Multi-pole permanent magnet synchronous generator wind turbines' grid support capability in uninterrupted operation during grid faults," IET Renewable Power Generation, vol. 3, no. 3, pp. 333-348, Sept. 2009.

[24] M. de prada Gil, "Design, operation and control of novel electrical concepts for offshore wind power plants", PhD. Thesis, Electr. Eng. Depart., Catalonia Institute for energy research, 2014.

[25] R. B. Cardenas and M. Molinas, "Comparative study of wind turbine power converters based on medium frequency ac-link for offshore dc grids", IEEE Journ. of Emer. and Selec. top. in Powe. Electron., vol. 3, no. 2, pp. 525-541, 2015.

[26] M. Hergt, D. Kurthakoti, and C. Schacherer, "Connecting power plants to high voltage networks", pat. U2 2016/0149509A1, 2016.

[27] IEEE Recommended Practice for $1 \mathrm{kV}$ to $35 \mathrm{kV}$ Medium-Voltage DC Power Systems on Ships," in IEEE Std 1709-2010, 2 Nov. 2010

[28] G. Li, J. Liang, S. Balasubramaniam, T. Joseph and C. E. Ugalde-Loo, "Frontiers of DC Circuit Breakers in HVDC and MVDC systems," in 1st IEEE Conference on Energy Internet and Energy System Integration, Beijing, China, Nov. 2017, pp. 1-6.

[29] M. Heidemann, G. Nikolic, A. Schnettler, A. Qawasmi, N. Soltau and R. W. De Donker, "Circuit-breakers for medium-voltage DC grids," in 2016 IEEE PES Transmission \& Distribution Conference and ExpositionLatin America (PES T\&D-LA), Morelia, 2016, pp. 1-6. 